\title{
INFECCIONES URINARIAS EN SITUACIONES ESPECIALES: TRASPLANTE RENAL Y DIÁLISIS
}

\author{
URINARY INFECTIONS IN SPECIAL SITUATIONS: \\ KIDNEY TRANSPLANT AND DIALYSIS
}

\author{
María José Pomares ${ }^{1}$
}

\begin{abstract}
RESUMEN
Los pacientes en hemodiálisis frecuentemente tienen más infecciones del tracto urinario (ITUs) debido al estado de inmunodepresión, la falta de remoción de gérmenes, la presencia de litiasis, vejiga neurogénica, anomalías en vía urinaria, uso de catéteres endovenosos y sondas vesicales. Los antibióticos empleados en el tratamiento de las ITUs en la población general pueden usarse en pacientes en hemodiálisis con el correspondiente ajuste de la dosis. La ITU es la infección bacteriana más común en los receptores de trasplante renal debido a factores como el tratamiento inmunosupresor, ITUs previas al trasplante, reflujo vesicoureteral, poliquistosis renal, diabetes, uso prolongado de catéteres urológicos, retraso en la función del injerto, episodios de rechazo agudo, bacteriuria asintomática a repetición y falta de profilaxis antibiótica. Se presentan principalmente en el postoperatorio inmediato y los primeros meses postrasplante. La repetición de las ITUs o episodios de bacteriuria asintomática pueden desencadenar rechazo agudo.
\end{abstract}

Palabras clave: insuficiencia renal crónica; piuria; hemodiálisis; nefropatía; trasplante renal; vejiga neurogénica.

Revista de la Sociedad Argentina de Diabetes 2019; Vol. 53 (28-50)

\begin{abstract}
Patients on hemodialysis often have more urinary tract infections (UTIS) due to immunosuppression, lack of germ removal, presence of lithiasis, neurogenic bladder, abnormalities in the urinary tract, use of intravenous catheters and urinary catheters. The antibiotics used in the treatment of UTIs in the general population can be used in hemodialysis patients with the corresponding adjustment of dose. UTI is the most common bacterial infection in kidney transplant recipients due to factors such as immunosuppressive therapy, UTIs prior to transplantation, vesicoureteral reflux, polycystic kidney disease, diabetes, prolonged use of urinary catheters, delay in graft function, episodes of acute rejection, recurrent asymptomatic bacteriuria and lack of antibiotic prophylaxis. They occur mainly in the immediate post-surgery period and the first months after transplant. The recurrence of UTIS or asymptomatic bacteriuria episodes can trigger acute rejection.
\end{abstract}

Key words: chronic renal insufficiency; pyuria; hemodialysis; nephropathy; kidney transplant; neurogenic bladder.

Revista de la Sociedad Argentina de Diabetes 2019; Vol. 53 (28-50)
Médica especialista en Nefrología y especializada en Diabetes, Hospital Penna, Consultorios del Centro de Enfermedades Renales e Hipertensión Arterial, Ciudad Autónoma de Buenos Aires, Argentina
Contacto de la autora: María José Pomares

E-mail: turcapomares@hotmail.com

Correspondencia: Beruti $28286^{\circ}$ piso, Depto. A (C1425BBH), Ciudad Autónoma de Buenos Aires, Argentina

Fecha de trabajo recibido: 25/03/19

Fecha de trabajo aceptado: 03/04/19

Conflictos de interés: la autora declara que no existe conflicto de interés
En vista del crecimiento sostenido de la diabetes mellitus (DM) a nivel mundial dado que la nefropatía por DM es la principal causa de insuficiencia renal crónica (IRC) ${ }^{1}$, será cada vez más habitual tratar pacientes con ambas patologías 2,3,4,5,6. En estos pacientes, a la mayor susceptibilidad a las infecciones y principalmente a las del tracto urinario determinada por la $\mathrm{DM}^{2,7,8}$, se suma la propia de la enfermedad renal y las distintas modalidades de terapia de reemplazo renal. Sin embargo existe poca literatura respecto de la infección del tracto urinario (ITU) en IRC ${ }^{4,9}$.

Las ITUs en pacientes en hemodiálisis (HD) son frecuentes por el estado de inmunodepresión por la uremia crónica, la pérdida de las propiedades antibacterianas de la orina, la falta de remoción de gérmenes por ausencia del flujo urinario, la presencia de litiasis, vejiga neurogénica, anomalías de la vía urinaria, uso de catéteres endovenosos y sondas vesicales comunes de observar en estos pacientes $^{9,10,11}$. Se presentan con mayor frecuencia complicaciones asociadas a un diagnóstico tardío dado que en pacientes con diuresis residual se observan manifestaciones similares a la población general pero en pacientes anúricos puede presentarse sólo con disconfort vesical y la imposibilidad de tomar muestras para urocultivo dificulta aún más el diagnóstico ${ }^{11}$. Los gérmenes más frecuentes no difieren de los observados en la población general, siendo la E. coli la más prevalente ${ }^{4,9,12,13}$. 
Cabe destacar el limitado valor diagnóstico de la presencia de piuria en estos pacientes dado que su ocurrencia en ausencia de infección es común en $\mathrm{HD}^{11,14}$ y podría asociarse al bajo flujo urinario, estasis vesical y en ocasiones a la enfermedad causante de la falla renal ${ }^{15}$. Sin embargo la presencia de infección sin piuria es poco común, por lo tanto la mayoría de los estudios coincide en el bajo valor predictivo positivo y el alto valor predictivo negativo de la piuria, y se sugiere la confirmación del diagnóstico con un urocultivo que además permita guiar el tratamiento 14,15,16,17.

Para el tratamiento de las ITUs se requieren altas concentraciones del antibiótico en orina y una concentración efectiva en el parénquima renal en caso de pielonefritis. Los antibióticos empleados para el tratamiento de las ITUs en la población general pueden emplearse en pacientes en HD con el correspondiente ajuste de la dosis, con excepción de la nitrofurantoína y el sulfametoxazol que se excretan muy poco en orina cuando cae el filtrado glomerular, no alcanzando una concentración urinaria terapéutica9 .

La ITU es la infección bacteriana más común en los receptores de trasplante renal ${ }^{18,19}$. El tratamiento inmunosupresor, las ITU previas al trasplante, el sexo femenino, el reflujo vesicoureteral, la poliquistosis renal, la DM, el uso prolongado de catéteres urológicos, el retraso en la función del injerto, los episodios de rechazo agudo, la bacteriuria asintomática (BA) a repetición y la falta de profilaxis antibiótica se asocian a mayor riesgo de ITU en esta población 18,19,20,21. La E. coli es el germen aislado con mayor frecuencia, y a las bacterias y hongos observados en la población general se suman gérmenes atípicos como micobacterias y virus $\mathrm{BK}^{18}$. Las ITUs se presentan principalmente en el postoperatorio inmediato y los primeros meses postrasplante coincidiendo con la mayor inmunosupresión. Estas ITUs tempranas tienen mayor tendencia a recurrir que las que se presentan en forma más tardía y con pronóstico más benevolente ${ }^{18,23,24}$.

La repetición de las ITUs o episodios de BA pueden desencadenar un episodio de rechazo agudo, lo que implica un tratamiento inmunosupresor más agresivo, un mayor riesgo de complicaciones infecciosas y en muchos casos tratamientos con esquemas nefrotóxicos por gérmenes resistentes $^{18,19,20,23}$. El impacto de las ITUs sobre el injerto respecto del rechazo crónico o disfunción no es concluyente, sin embargo la mayoría de los autores coincide que no se asocia a menor sobrevida del mismo, al menos en los casos de ITUs no recurrentes ${ }^{18,23}$. No obstante, debido a su gran frecuencia y posibles complicaciones con riesgo vital al tratarse de pacientes inmunodeprimidos, es necesario tomar medidas tendientes a su prevención. La rápida remoción de catéteres urológicos, evitar el uso rutinario de antimicrobianos a menos que existan manifestaciones clínicas de infección ${ }^{18,25}$, el manejo criterioso de los episodios de BA tratando sólo aquellos que se presentan en los primeros tres meses postrasplante o los que se asocian a un deterioro de la función del injerto ${ }^{22}$, reservar el tratamiento de la candiduria asintomática para los casos asociados a neutropenia o próxima intervención de la vía urinaria y la profilaxis antibiótica en los primeros tres a seis meses, son las principales medidas a tener en cuenta ${ }^{21}$.

El tratamiento de las ITUs en estos pacientes debe instaurarse rápidamente. La elección del esquema antibiótico se basa en la severidad de la infección, el antecedente de microorganismos resistentes y la sensibilidad en el antibiograma, recordando ajustar la dosis a la función renal y en casos de infección severa considerar disminuir a suspender la inmunosupresión 21,22.

\section{BIBLIOGRAFÍA}

1. Marinovich $S$, Lavorato $C$, Bisigniano $L$, et al. Registro argentino de diálisis crónica 2014-2015. Sociedad Argentina de Nefrología e Instituto Nacional Central Único Coordinador de Ablación e Implante. Buenos Aires 2016.

2. Nitzan O, Elias M , Chazan B, et al. Urinary tract infections in patients with type 2 diabetes mellitus: review of prevalence, diagnosis and management. Diabetes, Metabolic Syndrome and Obesity: Targets and Therapy 2015;8: 129-36.

3. Yu S, Fu A, Qiu Y, et al. Disease burden of urinary tract infections among type 2 diabetes mellitus patients in the US. J Diabetes Complication 2014; 28(5): 621-6.

4. Hsiao $C$, Lin $H$, Lin $Y$, et al. Urinary tract infection in patients with chronic kidney disease. Turk Journal of Medical Sciences 2014; 44(1): 145-9.

5. Nugent R, Fathima S, Feigl A, et al. The burden of chronic kidney disease on developing nations: a 21st century challenge in global health. Nephron Clin Pract 2011; 118(3): 269-77.

6. Levey A, Atkins R, Coresh J, et al. Chronic kidney disease as global public health problem: approaches and initiatives. A position statement from Kidney Disease Improving Global Outcomes. Kidney Int 2007; 72: 247-59.

7. Patterson J, Andriole V. Bacterial urinary tract infections in diabetes. Infect Dis Clin North Am 1997; 11(3): 735-50.

8. Shah B, Hux J. Quantifyting the risk of infectious diseases for people with diabetes. Diabetes Care 2003; 26(2): 510-13. 
9. Gilbert DN. Urinary Tract infections in patients with chronic renal insufficiency. Clin J Am Soc Nephrol 2006; 1(2): 327-31.

10. Tandogdu Z, Cai T, Koves B, et al. Urinary tract infections in inmunocompromised patients with diabetes, chronic kidney disease and kidney transplant. Eur Urol Focus 2016; 2(4): 394-99.

11. Falah $\mathrm{S}, \mathrm{Ammer} \mathrm{A}$, Kais $\mathrm{H}$. Urinary tract infection in hemodialysis patients with renal failure. J Fac Med Baghdad. 2012; 54(1): 38-41.

12. Ronald $A$. The etiology of urinary tract infection: traditional and emerging pathogens. Am J Med. 2002; 113 (Suppl 1A): S14-S19.

13. Hooton T. The current management strategies for community acquired urinary tract infection. Infect Dis Clin North Am 2003, 17(2): 303-32.

14. Vij R, Natarajav S, Peixoto A. Diagnostic utility of urinalysis in detecting urinary tract infection in hemodialysis patients. Nephron Clin Pract 2009; 113(4): 281-5.

15. Fasolo LR, Rocha LM, Campbell S, et al. Diagnostic relevance of pyuria in dialysis patients. Kidney International 2006; 70(11): 2035-38.

16. Oikonomou KG, Alhaddad A. The diagnostic value of urinalysis in hemodialysis patients with fever, sepsis or suspected urinary tract infection. Journal of Clinical and Diagnostic Research 2016; 10(10): 11-13.

17. Mortazavi M, Seyraian S, Shahidi S, et al. Pyuria as a screening test for detection of urinary tract infection in patients on long term hemodialysis. Iranian Journal of Kidney Disease 2011;5: 50-52.
18. Papasotiriou M, Savvidaki E, Kalliakmani P, et al. Predsiposing factors to the development of urinary tract infections in renal transplat recipients and the impact on the long term graft function. Renal Failure 2011; 33(4): 405-10.

19. Veroux M, Giuffrida G, Corona D, et al. Infective complications in renal allograft recipients: epidemiology and outcome. Transplant Proc 2008; 40(6): 1873-76.

20. Britt NS, Hagopian JC, Brennan DC, et al. Effects of recurrent urinary tract infections on graft and patient outcomes after kidney transplantation. Nephrol DialTransplant 2017; 32(10) 1758-66.

21. Vidal E, Cervera C, Cordero E, et al. Executive summary. Management of urinary tract infection in solid organ transplant recipients: consensus statementof the group for the study of infection in transplant recipients (GESITRA) of the Spanish Society of infectious disease and clinical microbiology (SEIMC) and the Spanish Network for research in infectious disease (REIPI). Enfern Infecc Microbiol Clin 2015; 33(10): 680-7.

22. Singh R, Geerlings SE, Bemelman FJ. Asymptomatic bacteriuria and urinary tract infections among renal allograft recipients. Curr Opin Infect Dis 2015; 28(1): 112-6.

23. Schmaldienst $S$, Dittrich $E$, Horl W, et al. Urinary tract infections after renal transplantation. Curr Opin Infect Dis 2002; 12(2): 125-30.

24. Di Cocco P, Orlano G, Mazzotta C, et al. Incidence of urinary tract infections caused by germs resistant to antibiotics commonly used after renal transplantation. Transplant Pro 2008; 40(6) 1881-4.

25. Renoult E, Aouragh F, Mayeux D, et al. Urinary tract infections during the 1st month after kidney transplantation. Agressologie $1992 ; 33(3)$ : $147-50$. 